\title{
Trazodone effects on developing brain
}

\author{
Zeljka Korade $\mathbb{C}^{1,2}$, Luke B. Allen $\mathbb{B}^{2,3}$, Allison Anderson ${ }^{3}$, Keri A. Tallman ${ }^{4}$, Thiago C. Genaro-Mattos ${ }^{3}$, Ned A. Porter ${ }^{4}$ and \\ Karoly Mirnics $\mathbb{1}^{2,3}$
}

\begin{abstract}
Trazodone (TRZ) is a commonly prescribed antidepressant with significant off-label use for insomnia. A recent drug screening revealed that TRZ interferes with sterol biosynthesis, causing elevated levels of sterol precursor 7-

dehydrocholesterol (7-DHC). Recognizing the well-documented, disruptive effect of 7-DHC on brain development, we designed a study to analyze TRZ effects during pregnancy. Utilizing an in vivo model and human biomaterial, our studies were designed to also account for drug interactions with maternal or offspring Dher 7 genotype. In a maternal exposure model, we found that TRZ treatment increased 7-DHC and decreased desmosterol levels in brain tissue in newborn pups. We also observed interactions between Dhcr7 mutations and maternal TRZ exposure, giving rise to the most elevated toxic oxysterols in brains of $D \mathrm{Cr}^{+/-}$pups with maternal TRZ exposure, independently of the maternal Dhcr7 genotype. Therefore, TRZ use during pregnancy might be a risk factor for in utero development of a neurodevelopmental disorder, especially when the unborn child is of $D H C R 7^{+/-}$genotype. The effects of TRZ on 7DHC was corroborated in human serum samples. We analyzed sterols and TRZ levels in individuals with TRZ prescriptions and found that circulating TRZ levels correlated highly with 7-DHC. The abundance of off-label use and high prescription rates of TRZ might represent a risk for the development of DHCR7 heterozygous fetuses. Thus, TRZ use during pregnancy is potentially a serious public health concern.
\end{abstract}

\section{Introduction}

The inhibition of 7-dehydrocholesterol reductase (DHCR7) enzyme, a key enzyme in the cholesterol biosynthesis pathway, leads to elevated levels of 7dehydrocholesterol (7-DHC) and decreased levels of desmosterol and cholesterol ${ }^{1}$. Inhibition of DHCR7 is a side effect of multiple widely-used pharmaceuticals ${ }^{2-4}$. These compounds produce changes in sterol profiles that mimic biochemical changes arising from genetic mutations in humans (Supplemental Fig. 1) ). $^{5,}$ One of these compounds is trazodone (TRZ) hydrochloride (marketed under brand names Trazodone, Desyrel, Donaren, Molipaxin, Oleptro, Trazorel, and Trittico).

\footnotetext{
Correspondence: Karoly Mirnics (karoly.mirnics@unmc.edu)

${ }^{1}$ Department of Pediatrics, College of Medicine, University of Nebraska Medical Center, Omaha 68198 NE, USA

2Department of Biochemistry and Molecular Biology, College of Medicine, University of Nebraska Medical Center, Omaha 68198 NE, USA

Full list of author information is available at the end of the article
}

Approved by the FDA in 1981 , TRZ is a potent $5-\mathrm{HT}_{2 \mathrm{~A}}$ and $\alpha_{1}$-adrenergic receptor antagonist and weak serotonin reuptake inhibitor ${ }^{7}$. TRZ and its major active metabolite meta-chlorophenylpiperazine ( $m$-CPP) also bind to a variety of other receptors. The primary use of TRZ is the treatment of depression. However, TRZ has been extensively prescribed for off-label use as a treatment for insomnia ${ }^{8,9}$. In fact, the off-label use of TRZ for insomnia has surpassed its usage as an antidepressant ${ }^{8}$. Nonapproved uses include treatment and/or self-treatment of opioid withdrawal symptom ${ }^{10}$, complex regional pain syndrome $^{11}$, obsessive-compulsive disorder ${ }^{12}$, alcohol withdrawal $^{13-15}$, schizophrenia ${ }^{16}$, dementia in Alzheimer's disease, eating disorders, fibromyalgia, and erectile dysfunction ${ }^{17}$. Considering this extensive off-label use, it is not surprising that for the period from 2007 to 2017 there were a total of $226,057,791$ TRZ prescriptions (Supplemental Fig. 2) ${ }^{18}$. The total number of TRZ prescriptions is almost twice as high as the number of oxycodone prescriptions. 
Normal cholesterol homeostasis is essential for brain development, health, and life ${ }^{19}$. Genetic disruptions of the cholesterol biosynthesis pathway lead to various syndromes, including Smith-Lemli-Opitz syndrome, desmosterolosis, and chondrodysplasia punctata X-linked 2 (CDPX2) $)^{5}$. Our recent study examined levels of cholesterol and cholesterol precursors, desmosterol, and 7-DHC in blood samples of 123 psychiatric patients treated with various antipsychotic and antidepressant drugs and 85 healthy controls ${ }^{20}$. We found markedly increased circulating 7-DHC levels in patients treated with TRZ, suggesting that TRZ is a strong inhibitor of $\mathrm{DHCR} 7^{21}$.

Knowing the well-documented, disruptive effect of 7DHC on brain development ${ }^{22-24}$, and the widespread offlabel use of TRZ in the human population, we designed a study to analyze TRZ effects during pregnancy using an in vivo model as well as a human biomaterial. Our studies were also designed to account for drug-genotype interactions, as we examined if the effects of TRZ were dependent on maternal or offspring $D h c r 7$ genotype $^{25}$. The study design is presented in Supplemental Fig. 3.

\section{Materials and methods Chemicals}

Unless otherwise noted, all chemicals were purchased from Sigma-Aldrich Co (St. Louis, MO). Highperformance liquid chromatography grade solvents were purchased from Thermo Fisher Scientific Inc. (Waltham, MA). TRZ was obtained from Sigma-Aldrich and dissolved in sterile DMSO solution for the experiments. All sterol standards, natural and isotopically labeled, used in this study are available from Kerafast, Inc. (Boston, MA).

\section{Trazodone injections in mice}

Adult male and female B6.129P2(Cg)-Dhcr $7^{\text {tm1Gst }} / \mathrm{J}$ stock \# 007453 mice were purchased from Jackson Laboratories. Mice homozygous for the $D h c r 7^{E x 8}$ allele lack the exon 8 coding sequence and flanking splice acceptor site of the targeted gene, resulting in the truncated DHCR7 mutation most frequently observed in SLOS patients (IVS8-1G >C). Homozygous mice die shortly after birth. Heterozygous $\mathrm{Dhcr} 7^{+/-}$mice are well, fertile, and indistinguishable from control, wild-type mice. Mice were maintained by breeding within the colony and refreshing twice a year with stock 000664 mice from Jackson Laboratories. Mice were housed under a $12 \mathrm{~h}$ light-dark cycle at constant temperature $\left(25^{\circ} \mathrm{C}\right)$ and humidity with ad libitum access to food (Teklad LM-485 Mouse/Rat Irradiated Diet 7912) and water in Comparative Medicine at the University of Nebraska Medical Center (UNMC), Omaha, NE. The time-pregnant female mice received $\mathrm{i} / \mathrm{p}$ injections of vehicle (5\% DMSO in saline) (VEH) or TRZ (20 mg/kg dissolved in VEH) from E12 to E19. In humans, TRZ (Desyrel) is given at a starting dose of $150 \mathrm{mg} /$ day; and may be increased by $50 \mathrm{mg}$ per day every 3-4 days to a maximum dose of $400 \mathrm{mg}$ per day for outpatient use. If we take a typical dose of $150 \mathrm{mg} / 70 \mathrm{~kg}$ human body weight, this translates to $2.1 \mathrm{mg} / \mathrm{kg} /$ day. Animal Equivalent dose (AED in $\mathrm{mg} / \mathrm{kg}$ ) is calculated as AED $(\mathrm{mg} / \mathrm{kg})=$ human dose $(\mathrm{mg} / \mathrm{kg})$ $(150 \mathrm{mg}$ per day $) \times \mathrm{Km}$ ratio $(12.3)=26.4 \mathrm{mg} / \mathrm{kg}^{26}$. As a result, we chose to use a low dose of $20 \mathrm{mg} / \mathrm{kg}$ in our mouse experiments, which translates back to $100-150 \mathrm{mg} /$ day in humans, depending on the weight of the patient. Twelve WT and $12 \mathrm{Dhcr}^{+/}$female mice were used in our study. Five WT and seven HET mice were injected with VEH and four WT and eight HET mice were injected with TRZ. The mouse colony was monitored three times a day and all newborn pups (P0) were collected for dissection shortly after birth. The total number of pups analyzed for this study was 160 (see Table 1 for detailed description). Frozen brain tissue samples were sonicated in ice-cold PBS containing butylated hydroxytoluene (BHT) and triphenylphosphine $\left(\mathrm{PPh}_{3}\right)$. The aliquots of homogenized tissue were used for sterol and oxysterol extractions and protein measurements. The

Table 1 Experimental groups.

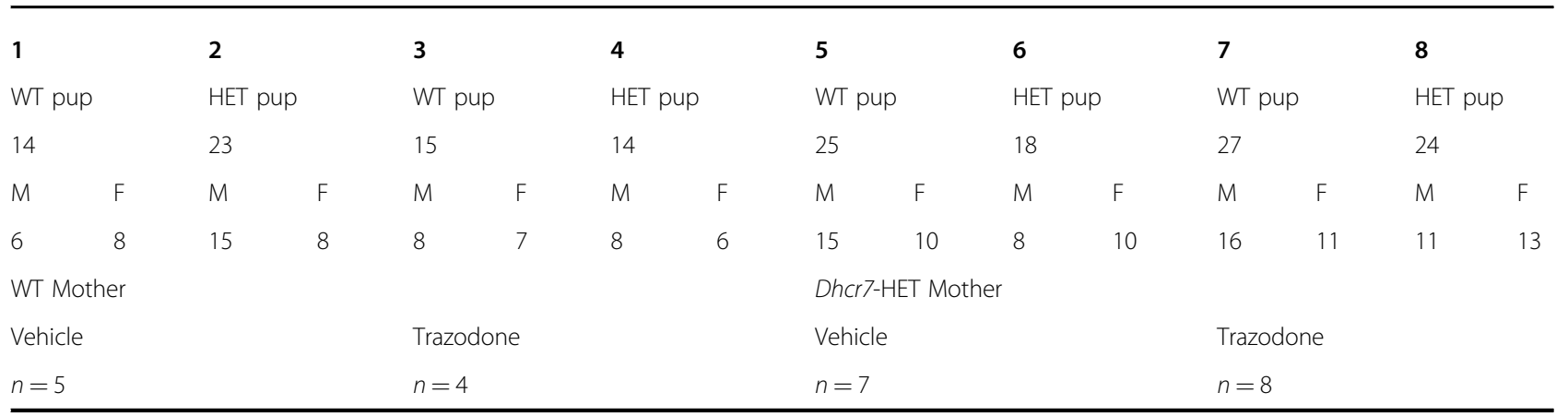

Groups 1-8 correspond to pups genotype. The numbers below WT and HET pups show how many pups were born and the next two rows show the distribution of male (M) and female (F) pups as determined by PCR. The pregnant mice were either WT or Dhcr7-HET. The numbers in the row below vehicle and TRZ show how many pregnant mice were used in the study. The total number of mice analyzed in this study were 160 pups from 12 vehicle-treated pregnant mice and 12 TRZ treated pregnant mice. 
protein was measured using a BCA assay (Pierce). Sterol levels were normalized to protein measurements and expressed as $\mathrm{nmol} / \mathrm{mg}$ protein. All procedures were performed in accordance with the Guide for the Humane Use and Care of Laboratory Animals. The use of mice in this study was approved by the Institutional Animal Care and Use Committee of UNMC.

\section{Liquid chromatography-mass spectrometry (LC-MS)/MS (SRM) analyses}

Sterols were extracted and derivatized with PTAD as described previously ${ }^{27}$ and placed in an Acquity UPLC system equipped with ANSI-compliant well plate holder coupled to a Thermo Scientific TSQ Quantis mass spectrometer equipped with an APCI source. Then $5 \mu \mathrm{L}$ was injected onto the column (Phenomenex Luna Omega C18, $1.6 \mu \mathrm{m}, 100 \AA$, $2.1 \mathrm{~mm} \times 50 \mathrm{~mm}$ ) with $100 \% \mathrm{MeOH}$ $(0.1 \% \mathrm{v} / \mathrm{v}$ acetic acid) mobile phase for 1.0 min runtime at a flow rate of $500 \mu \mathrm{L} / \mathrm{min}$. Natural sterols were analyzed by selective reaction monitoring (SRM) using the following transitions: Chol $369 \rightarrow 369,7-$ DHC $560 \rightarrow 365$, desmosterol $592 \rightarrow 560$, lanosterol $634 \rightarrow 602$, with retention times of $0.7,0.4,0.3$, and $0.3 \mathrm{~min}$, respectively. SRMs for the internal standards were set to $d_{7}-\mathrm{Chol}$ $376 \rightarrow 376, \quad d_{7}-7$-DHC $\quad 567 \rightarrow 372, \quad{ }^{13} \mathrm{C}_{3}$-desmosterol $595 \rightarrow 563,{ }^{13} \mathrm{C}_{3}$-lanosterol $637 \rightarrow 605$. Final sterol numbers are reported as $\mathrm{nmol} / \mathrm{mg}$ of protein.

\section{7-DHC- and cholesterol-derived oxysterol analysis}

7-DHC-derived oxysterols (DHCEO and 4 $\alpha-\mathrm{OH}-7$ $\mathrm{DHC}$ ) and cholesterol derived oxysterol 24-OH cholesterol were quantitated by LC-MS/MS using an APCI source in the positive ion mode. Lipid content from $200 \mu \mathrm{L}$ of brain lysate was extracted and the neutral lipids fraction was purified by SPE chromatography as described previously $y^{24}$. Purified content was resuspended in methanol and $5 \mu \mathrm{L}$ was injected onto the column (Phenomenex Luna Omega $\mathrm{C} 18,1.6 \mu \mathrm{m}, 100 \AA$, $2.1 \mathrm{~mm} \times$ $100 \mathrm{~mm})$ using ACN $(0.1 \% \mathrm{v} / \mathrm{v}$ acetic acid) (solvent $\mathrm{A})$ and methanol $(0.1 \% \mathrm{v} / \mathrm{v}$ acetic acid) (solvent B) as mobile phase. The gradient was: $5 \%$ B for 2 min; 5 to $95 \%$ B for $0.1 \mathrm{~min}$; $95 \%$ B for $1.5 \mathrm{~min}$; 95 to $5 \%$ B for $0.1 \mathrm{~min}$; $5 \%$ B for $0.5 \mathrm{~min}$. The oxysterols were analyzed by SRM using the following transitions: DHCEO $399 \rightarrow 381,4 \alpha-\mathrm{OH}-7-$ DHC $383 \rightarrow 365$, and 24-OH cholesterol $367 \rightarrow 367$. The SRM for the internal standard $\left(\mathrm{d}_{7}\right.$-chol) was set to $376 \rightarrow$ 376 and response factors were calculated to accurately determine the oxysterol levels. Final oxysterol levels are reported as $\mathrm{nmol} / \mathrm{mg}$ of protein.

\section{Human serum analysis}

The office of Regulatory Affairs has determined that this study does not constitute human subject research as defined at 45CFR46.102(f). Serum samples were obtained from the Nebraska Biobank that is part of the Center for Clinical and Translational Research. The biobank contains de-identified residual serum samples from patients who consent to donate any left-over after the laboratory testing. Electronic Health Record personnel identified a group of de-identified 59 samples: from users of TRZ (25 samples) and age, sex, and race matched control (34 samples) group. LC-MS/MS analyses were performed to detect and quantify TRZ and its active metabolite $m$ CPP in samples with TRZ listed in the medical records. Serum TRZ and $m$-CPP extraction and serum drug measurements were performed as previously described ${ }^{28}$. Serum sterol measurements by LC-MS/MS was done as previously described ${ }^{28}$.

\section{Statistical analyses}

Statistical analyses were performed using Graphpad Prism 8 for Windows. Data showed normal distribution, and the variance was comparable between the experimental and control groups. Unpaired two-tailed $t$ tests were performed for individual comparisons between the two groups. Welch's correction was employed when the variance between the two groups was significantly different. One-way ANOVA analyses were performed for comparisons among three or more groups. Two-way and threeway ANOVA analyses were performed to assess the interaction between maternal genotype, embryonic genotype, and drug treatment. The $\mathrm{p}$ values for statistically significant differences are highlighted in the figure legends.

\section{Results}

\section{Maternal TRZ exposure alters cholesterol biosynthesis in} the brain of offspring

WT and Dhcr7-HET pregnant mice received daily TRZ $(20 \mathrm{mg} / \mathrm{kg})$ or vehicle i/p injections from E12 to E19. This resulted in eight groups of newborn mice: (1) WT pupWT mother + vehicle; (2) WT pup-WT mother + TRZ; (3) HET pup-WT mother + vehicle; (4) HET pup -WT mother + TRZ; (5) WT pup-HET mother + vehicle; (6) WT pup-HET mother + TRZ; (7) HET pup-HET mother + vehicle; and (8) HET pup-HET mother + TRZ (Table 1). We hypothesized that the most pronounced effect would be observed in the HET pups from HET mothers treated with TRZ (group 8).

Brains were dissected from newborn pups and sterols were analyzed by LC-MS/MS. We report concentrations of cholesterol, desmosterol, 7-DHC, and lanosterol (Fig. 1) in WT and Dhcr $7^{+-}$pups born to either WT or Dhcr $7^{+/-}$ mothers. Maternal TRZ treatment, regardless of maternal or offspring Dhcr7 genotype, led to significantly elevated 7DHC in all groups $(\mathrm{p}<0.001)$. In contrast, desmosterol was significantly decreased by TRZ treatment. The effects of TRZ on cholesterol levels were much less pronounced, though $D h c r 7^{+/-}$pups were more affected by TRZ than 

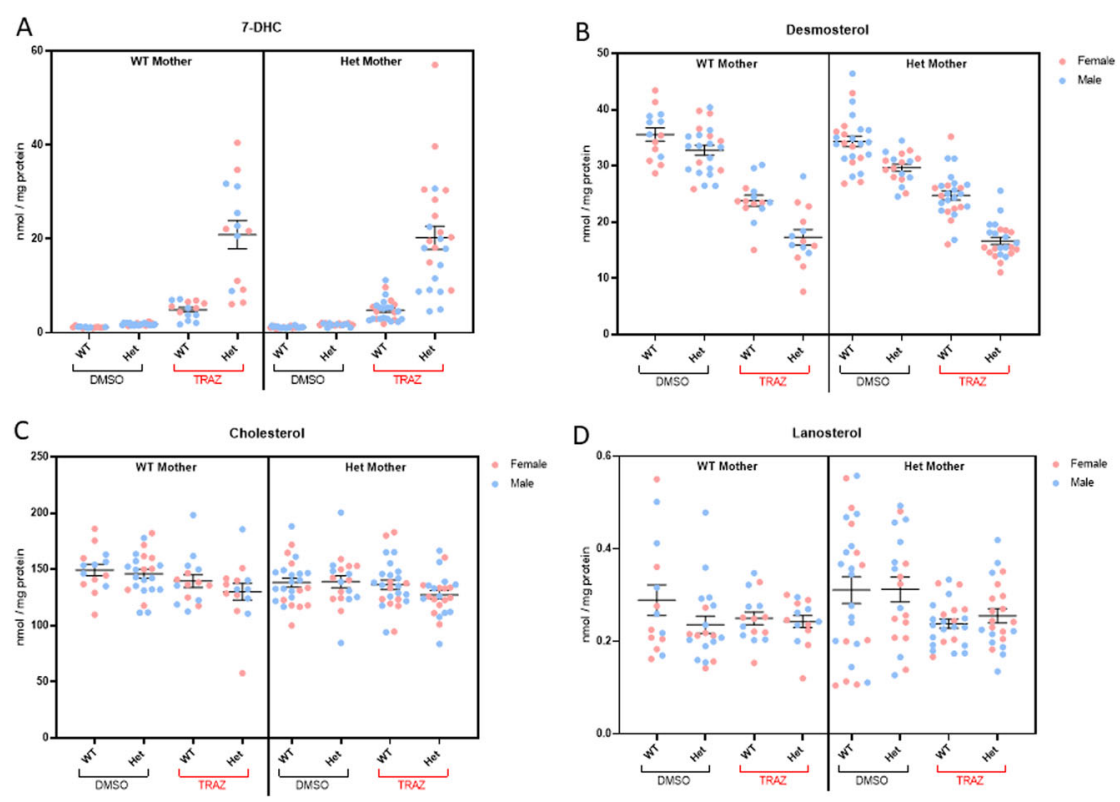

Fig. 1 Maternal exposure to TRZ during pregnancy alters sterol profile in the brains of newborn pups. Pregnant WT and Dhcr $7^{+/-}$females were exposed to TRZ from E12 to E19 and pups' brains were analyzed for sterols at P0. Each symbol corresponds to an individual pup brain; pink and blue symbols denote females and males, respectively. Bars correspond to the mean \pm SEM. A 7-DHC; B Desmosterol; C Cholesterol; D Lanosterol. Statistical analysis: Table 2.

Table 2 Three-way ANOVA of factors influencing brain sterol levels in newborn pups.

\begin{tabular}{|c|c|c|c|}
\hline ANOVA table & 7-DHC & Desmosterol & Cholesterol \\
\hline Trazodone treatment ${ }^{\mathrm{a}}$ & $P<0.0001$ & $P<0.0001$ & $P=0.005$ \\
\hline Maternal Dhcr7 genotype ${ }^{b}$ & $P=0.8237$ & $P=0.132$ & $P=0.0822$ \\
\hline Pup Dhcr7 genotype & $P<0.0001$ & $P<0.0001$ & $P=0.1316$ \\
\hline Trazodone * Maternal Dhcr7 genotype & $P=0.8676$ & $P=0.09$ & $P=0.3774$ \\
\hline Trazodone * Pup Dher7 genotype & $P<0.0001$ & $P=0.0093$ & $P=0.2518$ \\
\hline Maternal Dhcr7 genotype * Pup Dhcr7 genotype & $P=0.8658$ & $P=0.1991$ & $P=0.7382$ \\
\hline Trazodone * Maternal Dhcr7 genotype * Pup Dhcr7 genotype & $P=0.8966$ & $P=0.9108$ & $P=0.7932$ \\
\hline
\end{tabular}

${ }^{\mathrm{a} T R Z}$ versus DMSO alone.

${ }^{\mathrm{b}} \mathrm{WT}$ versus heterozygous for DHCR7.

Bold values indicates statistically significant.

their WT littermates. Maternal genotype by itself did not have an effect on the TRZ-induced sterol changes. For most conditions, there was no statistically significant difference between females and males. The exception was 7DHC where Dhcr $7^{+/}$male pups from $D h c r 7^{+/}$mothers were less affected than their female counterparts. Note that the greatest effects (and greatest variability) of TRZ on the sterol precursors were observed in the HET pups regardless of maternal genotype, suggesting a strong genetreatment interaction in the newborn brain. This variability could not be explained by pup sex, or mouse genetic background. However, 7-DHC levels were significantly correlated with TRZ levels. Thus, we believe that the source of this variability is related to maternal TRZ metabolism, which might depend on food intake, activity, or other factors. Three-way ANOVA results examining the variables of treatment, maternal genotype, and pup genotype are presented in Table 2 corresponding to the data in Fig. 1.

\section{Maternal TRZ exposure greatly elevates 7-DHC derived oxysterols in the brain of offspring}

The experiment above revealed that $D h c r 7^{+/-}$pups from $\mathrm{Dhcr} 7^{+/-}$mothers have about 12 times more 
7-DHC in the brain tissue than non-treated $D h c r 7^{+/-}$ pups. 7-DHC is a highly unstable molecule, and spontaneously generates toxic oxysterols ${ }^{22-24,29-31}$. To determine if this pronounced elevation of highly oxidizable 7-DHC leads to the formation of 7-DHC derived oxysterols, we measured $4 \alpha-\mathrm{OH}-\mathrm{DHC}$ and DHCEO in brain tissue (Fig. 2). Maternal TRZ treatment, regardless of maternal or offspring $D h c r 7$ genotype, led to significantly elevated 7-DHC-derived oxysterols in all groups receiving treatment. The largest increase in the level of oxysterols was observed in the brains of $D h c r 7^{+/}$pups. Of the two oxysterols, $4 \alpha-\mathrm{OH}-\mathrm{DHC}$ was present at the highest levels. In addition, we found a strong correlation between the levels of 7-DHC and

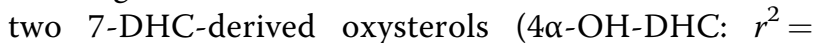
0.6362; $P<0.0001$; DHCEO: $r^{2}=0.4783 ; P<0.0001$ ). Three-way-ANOVA results examining the variables of treatment, maternal genotype, and pup genotype are presented in Table 3. In addition to 7-DHC-derived oxysterols, we assessed 24-OH-cholesterol levels derived from cholesterol. This oxysterol was not changed by TRZ treatment. The combined result show that the oxidative changes are primarily driven by 7 DHC levels (as opposed to cholesterol levels) and are most pronounced in the brains of maternally TRZtreated HET pups regardless of maternal genotype.

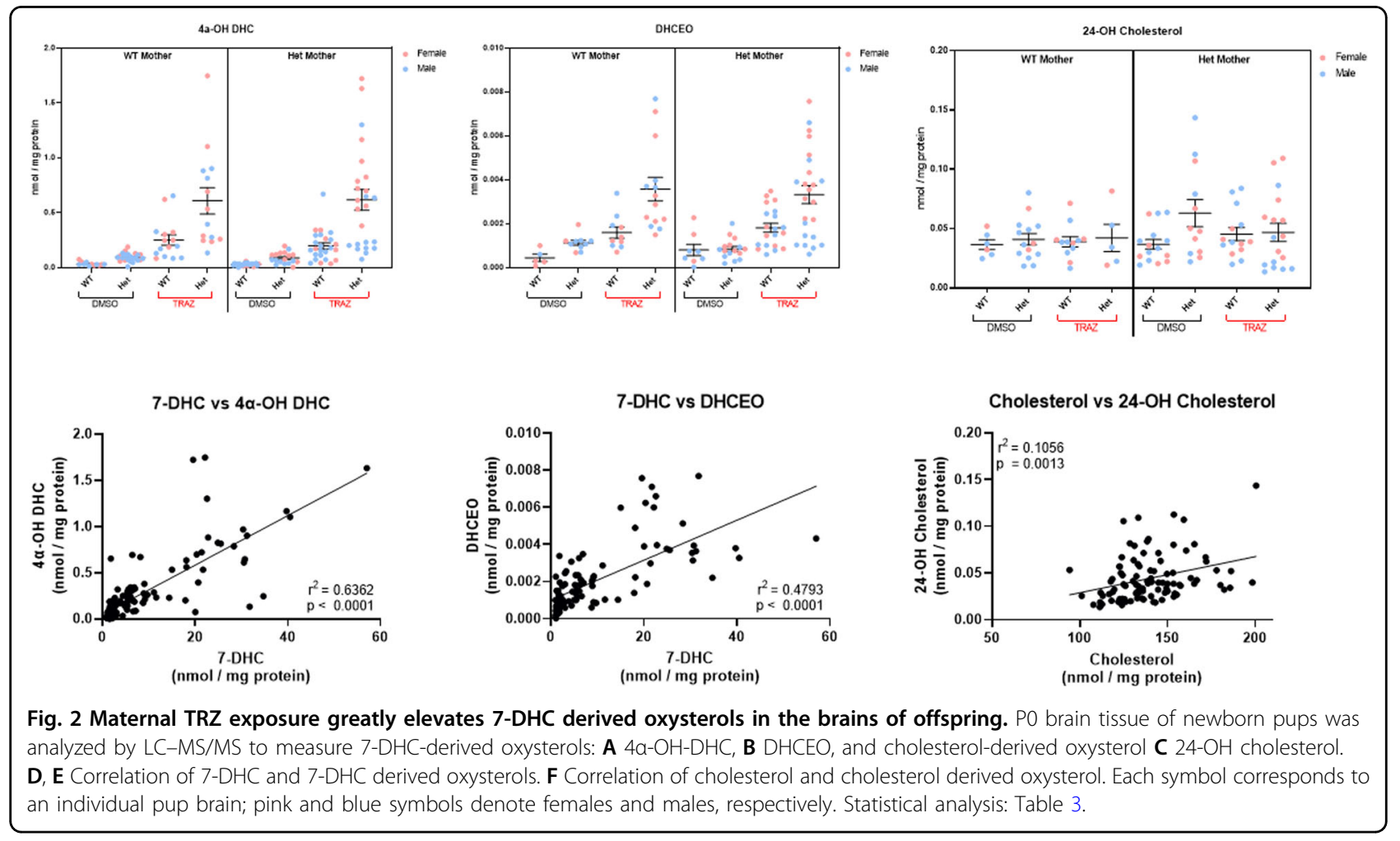

Table 3 Three-way ANOVA of factors influencing brain oxysterol levels in newborn pups.

\begin{tabular}{|c|c|c|}
\hline ANOVA table & $4 a-O H-D H C$ & DHCEO \\
\hline Trazodone treatment ${ }^{a}$ & $P<0.0001$ & $P<0.0001$ \\
\hline Maternal Dhcr7 genotype ${ }^{b}$ & $P=0.7238$ & $P=0.9649$ \\
\hline Pup Dhcr7 genotype ${ }^{b}$ & $P<0.0001$ & $P=0.0004$ \\
\hline Trazodone * Maternal Dhcr7 genotype & $P=0.8467$ & $P=0.9237$ \\
\hline Trazodone * Pup Dher7 genotype & $P<0.0001$ & $P=0.019$ \\
\hline Maternal Dhcr7 genotype * Pup Dhcr7 genotype & $P=0.7059$ & $P=0.3433$ \\
\hline Trazodone * Maternal Dhcr7 genotype * Pup Dhcr7 genotype & $P=0.7020$ & $P=0.8889$ \\
\hline
\end{tabular}

${ }^{\mathrm{a}} \mathrm{TRZ}$ versus DMSO alone.

${ }^{b}$ WT versus heterozygous for $D H C R 7$. 


\section{Circulating TRZ levels in human serum samples correlate with 7-DHC levels}

In our previous study, we showed that three drugs, aripiprazole, haloperidol, and TRZ, each increased circulating 7-DHC levels in blood samples of psychiatric patients $^{20}$. To corroborate and expand on our previous observations related to TRZ, we analyzed sterols and TRZ levels in serum samples from individuals with TRZ prescriptions. TRZ and its active metabolite $m$-CPP were analyzed in 25 new serum samples (Fig. 3A-D) and compared to sterol levels in 34 age- and sex-matched control samples. Sterol measurements revealed that TRZ greatly elevated circulating levels of $7-\mathrm{DHC}(P<0.001)$ and decreased desmosterol $(P<0.001)$, with a small effect on cholesterol and no effect on lanosterol (Fig. 4A-D; Supplemental Fig. 4A-D male vs. females). The circulating TRZ levels correlated highly with 7-DHC $\left(r^{2}=0.3887\right.$; $P=0.0009$ ) (Fig. 5). The outcome in this serum assessment suggests that TRZ-driven cholesterol biosynthesis changes in humans likely occur on a similar scale and by the same mechanisms as in our in vivo model and that it is likely occurring in multiple tissues outside the brain.

\section{Discussion}

The key outcomes of our studies can be summarized as follows: (1) TRZ exposure increases 7-DHC, and decreases desmosterol levels in an in vivo rodent model. (2) Dhcr 7 mutations and maternal TRZ exposure interact, giving rise to toxic oxysterols that are disruptive to brain development. (3) The brains of $D h c r 7^{+/-}$pups show the greatest disruptions of sterol profile in response to maternal TRZ exposure, and this does not depend on maternal Dhcr7 genotype. (4) TRZ exposure has a strong effect on circulating sterol levels in individuals taking TRZ. On a population scale, our findings point to a potentially serious public health impact of a medication as common as TRZ.

Elevation of 7-DHC concomitant with a decrease in desmosterol is characteristic for the inhibition of the DHCR7 enzyme (Supplemental Fig. 1). DHCR7 converts 7-DHC to cholesterol, and 7-dehydrodesmosterol into desmosterol. This accumulation of precursors has biological consequences, even when the difference is only a single double bond between the molecular structures ${ }^{32}$. 7DHC is the most oxidizable (and unstable) sterol with a peroxidation rate constant of 2160 , about 200 -fold more than cholesterol and 10 times more than arachidonic acid, which is generally considered to be highly oxidizable ${ }^{33}$. As a result, 7-DHC readily gives rise to 7-DHC derived oxysterols $^{30}$ that are well-described and their biological function has been extensively studied ${ }^{22-24,30,31,34}$. We have shown that 7-DHC derived oxysterols have potent and detrimental biological effects on cell proliferation, gene expression, and neuronal arborization ${ }^{22-24,30,31,34}$.

Over the last decade, many psychotropic drugs have been shown to interfere with sterol biosynthesis,

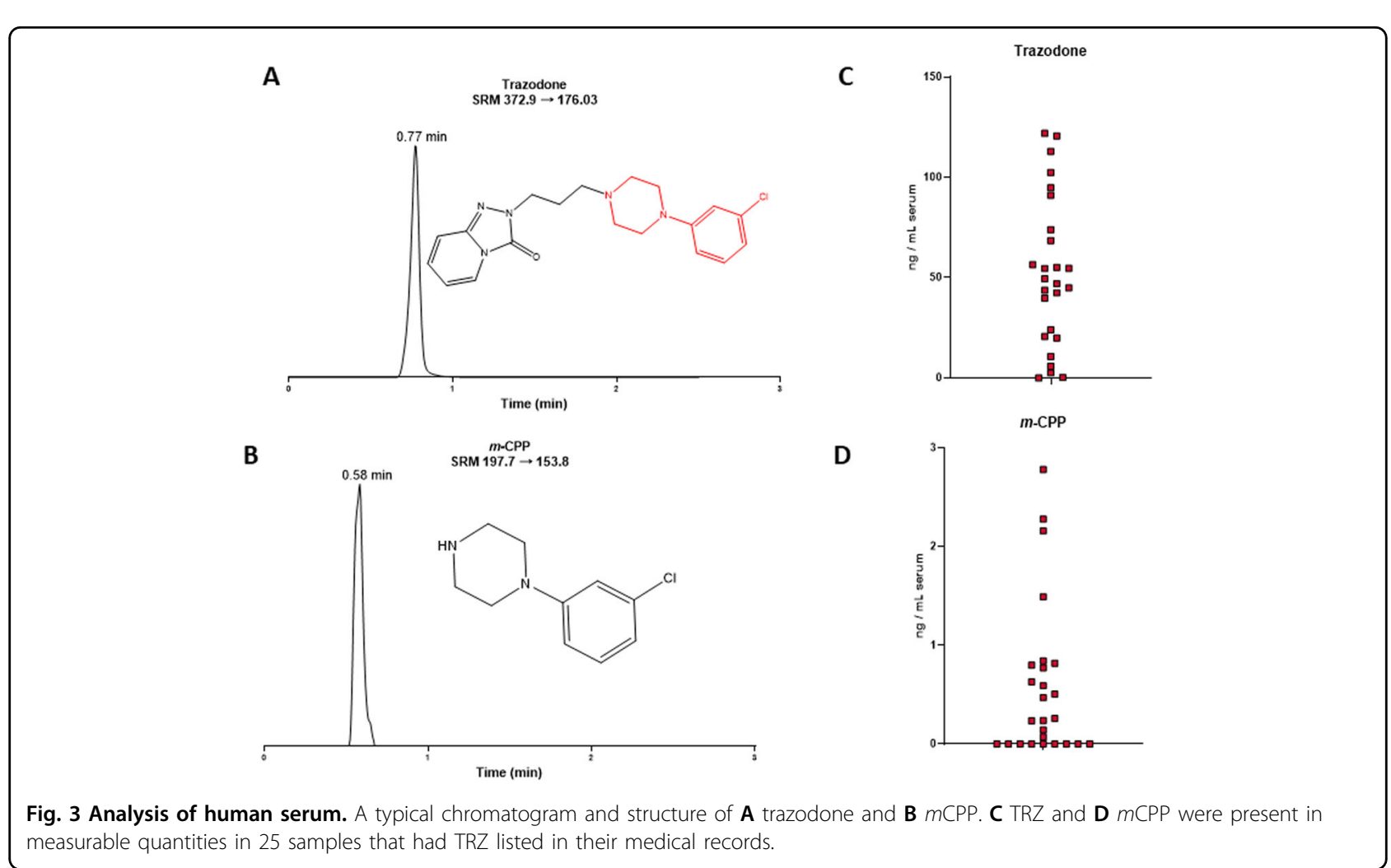



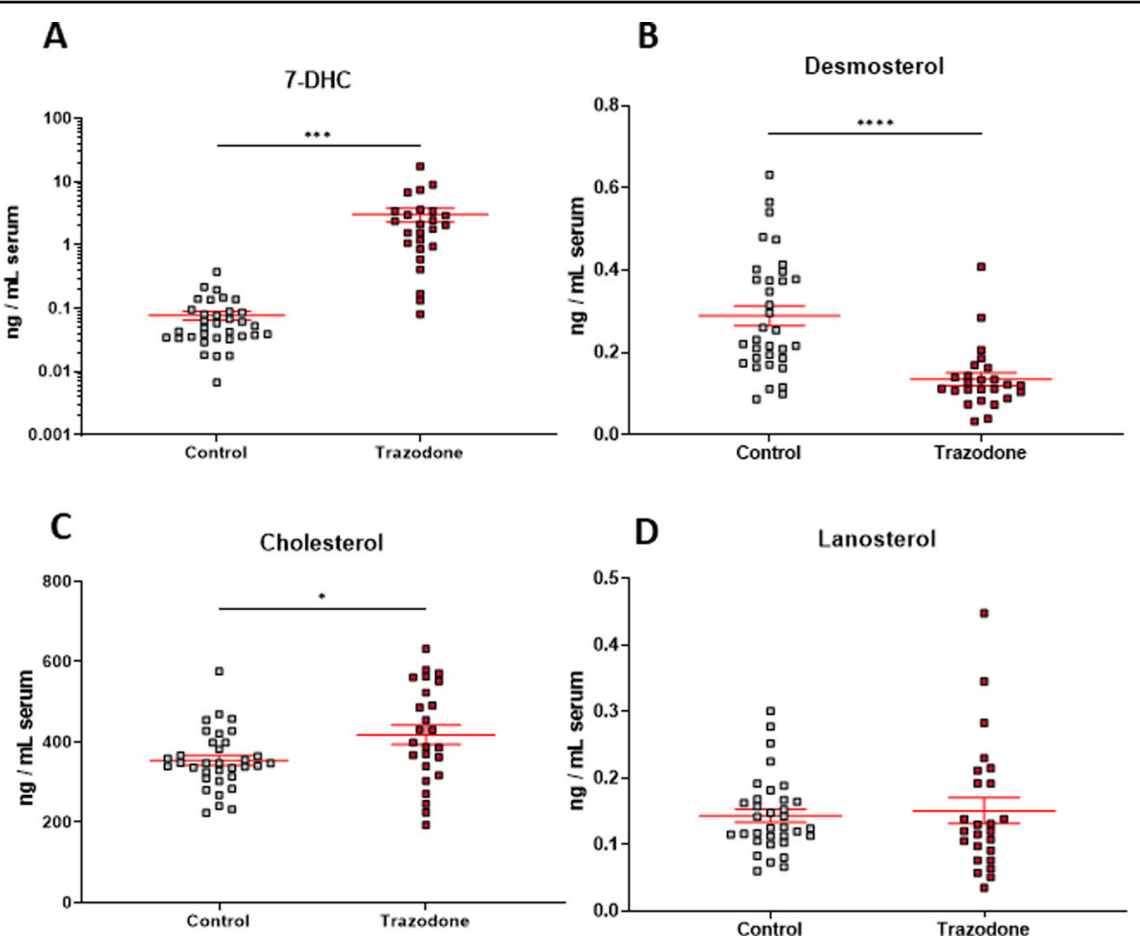

Fig. 4 Sterol content in human serum. A 7-DHC, B desmosterol, C cholesterol, and $\mathbf{D}$ lanosterol were measured in serum samples from TRZ-treated individuals or controls. Note logarithmic $y$-axis to allow that all samples are visible as individual dots.

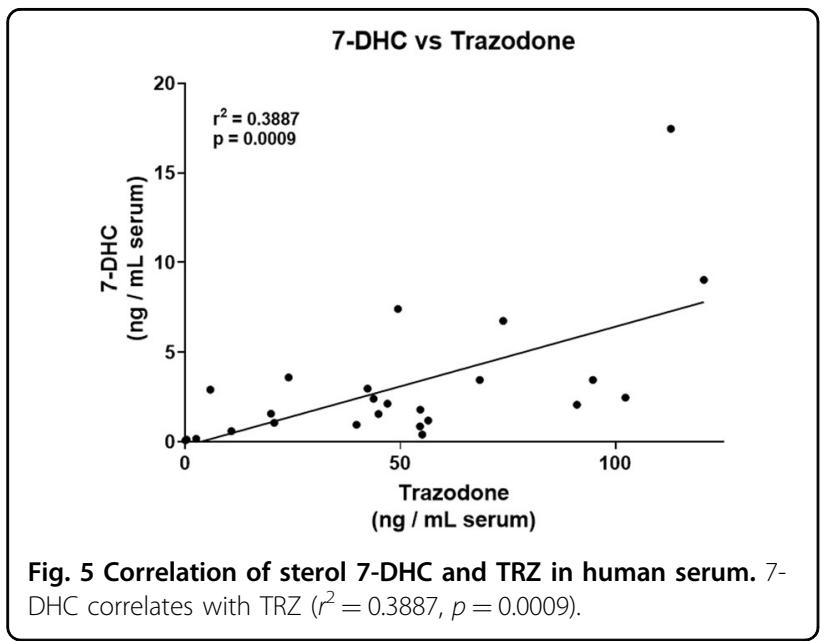

increasing 7-DHC and resulting in oxysterol levels ${ }^{2-4}$. These properties were described initially in experiments with cell lines, followed by validation in animal models and human blood samples. Cariprazine ${ }^{27,35}$, haloperidol ${ }^{20}$, aripiprazole ${ }^{36}$, and TRZ are only a few examples of these DHCR7 inhibiting compounds. However, it should be noted that many other approved, non-CNS medications also show similar sterol-interfering effects, including amiodarone ${ }^{28}$. This raises an important question: which patient populations are most sensitive to the side effects of sterol-interfering drugs? Multiple animal studies to date suggest that two populations might be of risk-a developing embryo, and individuals with a single allele mutation in the DCHR7 gene. In addition, it appears that the risk is cumulative. In embryonic development, drug exposure and DHCR7 genotype interact, elevating 7-DHC to levels seen in genetic mouse models of SLOS. This brings us to the next question: the human relevance of our and others' findings.

There is a legitimate concern that the inhibition of DHCR7 by TRZ in the developing human brain could have severe adverse effects. We base this view on the data from human genetic syndrome SLOS where mutations in DHCR7 affect CNS structure and function leading to developmental disabilities and autism ${ }^{37,38}$. Biochemical data from SLOS patient biomaterials suggests that TRZ could have teratogenic effects and should be avoided during pregnancy, especially if the fetus is heterozygous for DCHR7. Finally, a comprehensive literature review has identified that exposure to DHCR7 inhibitors during pregnancy leads to fetal loss in humans ${ }^{39}$, similar to those of known teratogens.

The public health relevance of our findings is also important to consider. Heterozygosity in DHCR7 is quite common in the human population, with estimates ranging from 1 to $1.5 \%{ }^{40}$, and it is not routinely tested for. Combined with the abundance of off-label use and high prescription rates, maternal TRZ exposure in DHCR7 heterozygous 
fetuses might result in altered development, leading to developmental disability or other long-term consequences. It should be also considered that TRZ tends to be used in combination with other antipsychotics and pharmaceuticals that by themselves also inhibit sterol synthesis. The summative effect of this polypharmacy is unknown, but could be a significant concern: sterol homeostasis is undoubtedly essential for brain development and function, and interference with this finely tuned, intrinsically regulated system should be studied more extensively. Similarly, sterol biosynthesis disruption could have detrimental effects on other body systems, as sterols are essential precursors of many critical molecules-from steroid hormones to vitamins ${ }^{1,5}$.

\section{Acknowledgements}

This work was supported by the National Institutes of Health NIMH R01 MH1 10636 (K.M. and N.A.P.), R01 MH067234 (K.M.), and NICHD HD064727 (N. A.P.). The query of the Nebraska Biobank of de-identified electronic health records has been subsidized by the Great Plains IDeA-CTR grant NIH U54GM115458/NIGMS NIH. Special thanks to the participants who opt to donate their left-over serum samples to Nebraska Biobank and to Dr. Guda Purnima and Neeharica Kodali for the search of Electronic Health Records. The authors would like to thank Dr. Matthew R. Sandbulte for editing the text.

\section{Author details}

'Department of Pediatrics, College of Medicine, University of Nebraska Medical Center, Omaha 68198 NE, USA. ${ }^{2}$ Department of Biochemistry and Molecular Biology, College of Medicine, University of Nebraska Medical Center, Omaha 68198 NE, USA. ${ }^{3}$ Munroe-Meyer Institute for Genetics and Rehabilitation, University of Nebraska Medical Center, Omaha 68105 NE, USA. ${ }^{4}$ Department of Chemistry, Vanderbilt Institute of Chemical Biology and Vanderbilt Kennedy Center for Research on Human Development, Vanderbilt University, Nashville 37235 TN, USA

\section{Author contributions}

K.M. and N.A.P. conceived the study, obtained the funding, and designed the experiments; Z.K., L.A., A.A., and T.M. performed the animal experiments, laboratory, and statistical analysis; K.T. synthesized all sterol and oxysterol standards; all authors edited and approved the final version.

\section{Conflict of interest}

The authors declare that they have no conflict of interest.

\section{Publisher's note}

Springer Nature remains neutral with regard to jurisdictional claims in published maps and institutional affiliations.

Supplementary information The online version contains supplementary material available at https://doi.org/10.1038/s41398-021-01217-w.

Received: 6 September 2020 Revised: 6 January 2021 Accepted: 14 January 2021

Published online: 01 February 2021

\section{References}

1. Porter, F. D. RSH/Smith-Lemli-Opitz syndrome: a multiple congenital anomaly/ mental retardation syndrome due to an inborn error of cholesterol biosynthesis. Mol. Genet. Metab. 71, 163-174 (2000).

2. Kim, H. Y. et al. Inhibitors of 7-dehydrocholesterol reductase: screening of a collection of pharmacologically active compounds in neuro2a cells. Chem. Res. Toxicol. 29, 892-900 (2016)
3. Korade, Z. et al. The effect of small molecules on sterol homeostasis: measuring 7-dehydrocholesterol in Dhcr7-deficient neuro2a cells and human fibroblasts. J. Med. Chem. 59, 1102-1115 (2016).

4. Wages, P. A., Kim, H. H., Korade, Z. \& Porter, N. A. Identification and characterization of prescription drugs that change levels of 7-dehydrocholesterol and desmosterol. J. Lipid Res. 59, 1916-1926 (2018).

5. Porter, F. D. \& Herman, G. E. Malformation syndromes caused by disorders of cholesterol synthesis. J. Lipid Res. 52, 6-34 (2011).

6. Honda, A. et al. Highly sensitive analysis of sterol profiles in human serum by LC-ESI-MS/MS. J. Lipid Res. 49, 2063-2073 (2008).

7. Fagiolini, A., Comandini, A., Catena Dell'Osso, M. \& Kasper, S. Rediscovering trazodone for the treatment of major depressive disorder. CNS Drugs $\mathbf{2 6}$, 1033-1049 (2012).

8. Jaffer, K. Y. et al. Trazodone for insomnia: a systematic review. Innov. Clin. Neurosci. 14, 24-34 (2017).

9. La, A. L. et al. Long-term trazodone use and cognition: a potential therapeutic role for slow-wave sleep enhancers. J. Alzheimers Dis. 67, 911-921 (2019).

10. Kurtz, S. P., Buttram, M. E., Margolin, Z. R. \& Wogenstahl, K. The diversion of nonscheduled psychoactive prescription medications in the United States, 2002 to 2017. Pharmacoepidemiol. Drug Saf. 28, 700-706 (2019).

11. Belinskaia, D. A., Belinskaia, M. A., Barygin, O. I., Vanchakova, N. P. \& Shestakova, N. N. Psychotropic drugs for the management of chronic pain and itch. Pharmaceuticals 12, 99, https://doi.org/10.3390/ph12020099 (2019).

12. Prasad, A. Efficacy of trazodone as an anti obsessional agent. Pharm. Biochem. Behav. 22, 347-348 (1985).

13. Borras, L., de Timary, P., Constant, E. L., Huguelet, P. \& Eytan, A. Successful treatment of alcohol withdrawal with trazodone. Pharmacopsychiatry 39, 232 (2006).

14. Le Bon, O. et al. Double-blind, placebo-controlled study of the efficacy of trazodone in alcohol post-withdrawal syndrome: polysomnographic and clinical evaluations. J. Clin. Psychopharmacol. 23, 377-383 (2003).

15. Roccatagliata, G., Albano, C., Maffini, M. \& Farelli, S. Alcohol withdrawal syndrome: treatment with trazodone. Int. Pharmacopsychiatry 15, 105-110 (1980).

16. Singh, S. P., Singh, V., Kar, N. \& Chan, K. Efficacy of antidepressants in treating the negative symptoms of chronic schizophrenia: meta-analysis. Br. J. Psychiatry 197, 174-179 (2010).

17. Fink, H. A., MacDonald, R., Rutks, I. R. \& Wilt, T. J. Trazodone for erectile dysfunction: a systematic review and meta-analysis. BJU Int. 92, 441-446 (2003).

18. Drug Stat. Trazodone Hydrochloride Yearly Prescription. https://lincalc.com/ DrugStats/Drugs/TrazodoneHydrochloride (2021).

19. Korade, Z. \& Kenworthy, A. K. Lipid rafts, cholesterol, and the brain. Neuropharmacology 55, 1265-1273 (2008).

20. Korade, Z. et al. Effect of psychotropic drug treatment on sterol metabolism. Schizophr. Res. 187, 74-81 (2017).

21. Hall, P. et al. Aripiprazole and trazodone cause elevations of 7dehydrocholesterol in the absence of Smith-Lemli-Opitz Syndrome. Mol. Genet. Metab. 110, 176-178 (2013).

22. Korade, Z., Xu, L., Mirnics, K. \& Porter, N. A. Lipid biomarkers of oxidative stress in a genetic mouse model of Smith-Lemli-Opitz syndrome. J. Inherit. Metab. Dis. 36, 113-122 (2013).

23. Korade, Z., Xu, L., Shelton, R. \& Porter, N. A. Biological activities of 7dehydrocholesterol-derived oxysterols: implications for Smith-Lemli-Opitz syndrome. J. Lipid Res. 51, 3259-3269 (2010).

24. $\mathrm{Xu}$, L. et al. DHCEO accumulation is a critical mediator of pathophysiology in a Smith-Lemli-Opitz syndrome model. Neurobiol. Dis. 45, 923-929 (2012).

25. Korade, Z. et al. Vulnerability of $\mathrm{DHCR7}(+/-)$ mutation carriers to aripiprazole and trazodone exposure. J. Lipid Res. 58, 2139-2146 (2017).

26. Nair, A. B. \& Jacob, S. A simple practice guide for dose conversion between animals and human. J. Basic Clin. Pharm. 7, 27-31 (2016).

27. Genaro-Mattos, T. C. et al. Dichlorophenyl piperazines, including a recentlyapproved atypical antipsychotic, are potent inhibitors of DHCR7, the last enzyme in cholesterol biosynthesis. Toxicol. Appl. Pharm. 349, 21-28 (2018).

28. Allen, L. B. et al. Amiodarone alters cholesterol biosynthesis through tissuedependent inhibition of emopamil binding protein and dehydrocholesterol reductase 24. ACS Chem. Neurosci. 11, 1413-1423 (2020).

29. Korade, Z. et al. Antioxidant supplementation ameliorates molecular deficits in Smith-Lemli-Opitz syndrome. Biol. Psychiatry 75, 215-222 (2014).

30. Xu, L., Korade, Z. \& Porter, N. A. Oxysterols from free radical chain oxidation of 7-dehydrocholesterol: product and mechanistic studies. J. Am. Chem. Soc. 132 2222-2232 (2010). 
31. $\mathrm{Xu}$, L. et al. An oxysterol biomarker for 7-dehydrocholesterol oxidation in cell/ mouse models for Smith-Lemli-Opitz syndrome. J. Lipid Res. 52, 1222-1233 (2011).

32. $\mathrm{Xu}, \mathrm{L}$. \& Porter, N. A. Free radical oxidation of cholesterol and its precursors: Implications in cholesterol biosynthesis disorders. Free Radic. Res. 49, 835-849 (2015).

33. Lamberson, C. R. et al. Propagation rate constants for the peroxidation of sterols on the biosynthetic pathway to cholesterol. Chem. Phys. Lipids 207, 51-58 (2017).

34. Xu, L., Korade, Z., Rosado, D. A. Jr., Mirnics, K. \& Porter, N. A. Metabolism of oxysterols derived from nonenzymatic oxidation of 7-dehydrocholesterol in cells. J. Lipid Res. 54, 1135-1143 (2013).

35. Genaro-Mattos, T. C. et al. Maternal cariprazine exposure inhibits embryonic and postnatal brain cholesterol biosynthesis. Mol. Psychiatry 25, 2685-2694, https://doi.org/10.1038/s41380-020-0801-x (2020).
36. Genaro-Mattos, T. C. et al. Maternal aripiprazole exposure interacts with 7dehydrocholesterol reductase mutations and alters embryonic neurodevelopment. Mol. Psychiatry 24, 491-500 (2019).

37. Bukelis, I., Porter, F. D., Zimmerman, A. W. \& Tierney, E. Smith-Lemli-Opitz syndrome and autism spectrum disorder. Am. J. Psychiatry 164, 1655-1661 (2007).

38. Nowaczyk, M. J. \& Irons, M. B. Smith-Lemli-Opitz syndrome: phenotype, natural history, and epidemiology. Am. J. Med Genet. C Semin. Med. Genet. 160C 250-262 (2012).

39. Boland, M. R. \& Tatonetti, N. P. Investigation of 7-dehydrocholesterol reductase pathway to elucidate off-target prenatal effects of pharmaceuticals: a systematic review. Pharmacogenomics J. 16, 411-429 (2016).

40. Cross, J. L. et al. Determination of the allelic frequency in Smith-Lemli-Opitz syndrome by analysis of massively parallel sequencing data sets. Clin. Genet. 87, 570-575 (2015). 